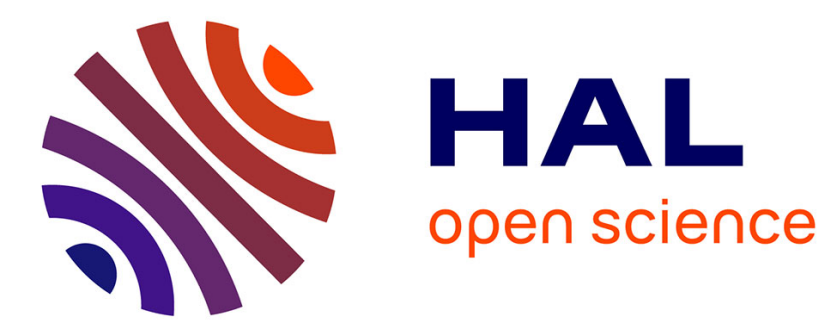

\title{
L'influence des auditeurs externes et des comités d'audit sur le contenu informatif des manipulations comptables
}

Rémi Janin, Charles Piot

\section{To cite this version:}

Rémi Janin, Charles Piot. L'influence des auditeurs externes et des comités d'audit sur le contenu informatif des manipulations comptables. LA COMPTABILITE, LE CONTRÔLE ET L'AUDIT ENTRE CHANGEMENT ET STABILITE, May 2008, France. pp.CD Rom. halshs-00525377

\section{HAL Id: halshs-00525377 \\ https://shs.hal.science/halshs-00525377}

Submitted on 11 Oct 2010

HAL is a multi-disciplinary open access archive for the deposit and dissemination of scientific research documents, whether they are published or not. The documents may come from teaching and research institutions in France or abroad, or from public or private research centers.
L'archive ouverte pluridisciplinaire HAL, est destinée au dépôt et à la diffusion de documents scientifiques de niveau recherche, publiés ou non, émanant des établissements d'enseignement et de recherche français ou étrangers, des laboratoires publics ou privés. 


\title{
L'influence des auditeurs externes et des comités d'audit sur le contenu informatif des manipulations comptables
}

\section{The Effects of External Auditors and Audit Commitees on the Informativeness of Earnings}

\author{
Rémi Janin \\ Membre du CERAG-UMR 5820 ; Maître de conférences à l'IUT 2 \\ 1 Place de Verdun, 38000 Grenoble \\ Professeur affilié à Grenoble Ecole de Management \\ Tél. : 0476037959 \\ Fax : 0476513547 \\ remi.janin@iut2.upmf-grenoble.fr \\ Charles Piot \\ Membre du CERAG-UMR 5820 ; Professeur à l'IAE de Grenoble, \\ 150 rue de la Chimie, BP 47, 38040 Grenoble cedex 09 \\ Tél. : 0476635370 \\ Fax : 0476546068 \\ Charles.piot@upmf-grenoble.fr
}

Résumé. La flexibilité des règles comptables permet aux dirigeants d'exercer leur jugement pour publier des résultats en fonction d'objectifs discrétionnaires. Or, même si l'on considère que les investisseurs ont une lecture relativement sophistiquée des états financiers, il n'est pas aisé de distinguer les manipulations comptables opportunistes, trompeuses, de celles qui, au contraire, permettent aux dirigeants de renforcer le contenu informatif des chiffres comptables. Si l'audit a alors un rôle privilégié à jouer, nous postulons que la capacité d'un audit de qualité à renforcer la pertinence des chiffres comptables est contingente au cadre normatif utilisé. Dans le contexte normatif français, cette étude teste alors l'impact de divers attributs de qualité du processus d'audit (réputation et ancienneté de l'auditeur, présence d'un comité d'audit indépendant) sur le contenu informatif des régularisations comptables (accruals) discrétionnaires. L'échantillon comprend 152 firmes-année du SBF120 sur la période 1999-2001. Les résultats indiquent, en contraste avec certains travaux américains, que le contenu informatif des accruals discrétionnaires est moindre en présence d'un auditeur appartenant aux Big N, ou en présence d'un comité d'audit en majorité indépendant. La simple présence d'un comité d'audit, peu importe son degré d'indépendance, n'altère pas significativement le contenu informatif des accruals discrétionnaires, tout comme l'existence d'une longue relation entre l'auditeur et son client.

Mots clés. Accruals, contenu informatif, qualité de l'audit, étude d'association.

\begin{abstract}
Because of the flexibility allowed by accounting standards, accrual-based accounting is subject to managerial discretion. Although one may consider that investors are sophisticated enough to read and understand financial reports, it is difficult to find out whether discretionary accounting accruals are opportunistic, thus foolish and value irrelevant, or whether managerial discretion enhances earnings informativeness by allowing the communication of private information about firm value. If auditing can play an important role in improving the credibility of reported earnings, we argue that the impact of audit quality on the value relevance of accounting numbers is contingent to the accounting framework used. Using French accounting standards, this paper examines whether various audit quality attributes (auditor reputation, auditor tenure, existence of an independent audit committee) have an impact on the value relevance of discretionary accruals. The sample is composed of 152 firm-year observations from the SBF 120 Index during 1999-2001. In contrast with some US studies, empirical findings document that the value relevance of discretionary accruals is lower if the firm appoints a Big $\mathrm{N}$ auditor, or if an audit committee in majority independent exists. However, the only existence of an audit committee, regardless of its independence, has no effects on the value relevance of discretionary accruals, nor does long auditor tenure.
\end{abstract}

Key words. Accruals, value relevance, audit quality, association study. 


\section{Introduction}

En dépit des nombreux scandales financiers de ces dernières années, les états financiers publiés par les entreprises demeurent pour les investisseurs une source d'information privilégiée. À cet égard, les normes IAS/IFRS accordent une place centrale aux investisseurs parmi les utilisateurs de la comptabilitéi. Il est cependant avéré que la flexibilité des règles comptables permet aux dirigeants d'exercer leur jugement pour publier des résultats en fonction d'objectifs discrétionnaires. Contrairement à la plupart des études publiées à ce sujet, dans cet article nous intéressons aux motivations vertueuses des dirigeants en la matière. En effet, si l'on considère que les dirigeants sont mieux informés que les investisseurs sur les perspectives de leur entreprise, ils ont intérêt, dans un but de signalisation, à communiquer au marché, par une gestion adéquate du résultat comptable, l'information privée qu'ils détiennent. Or, même si la plupart des investisseurs ont une lecture relativement sophistiquée des états financiers, il n'est pas aisé de distinguer si les dirigeants gèrent de façon opportuniste et donc trompeuse les chiffres qu'ils publient, ou si, au contraire, ils visent par leur intervention à renforcer le contenu informatif des chiffres comptables afin de mieux informer le marché sur les perspectives de la firme. L'audit a alors un rôle privilégié à jouer. En certifiant l'information publiée, les auditeurs externes engagent leur responsabilité et, par là même, contribuent à renforcer la confiance de ses utilisateurs. Les résultats de Subramanyan (1996) suggèrent que les dirigeants usent de leur pouvoir discrétionnaire pour renforcer le contenu informatif des résultats qu'ils publient. Krishnan (2003) montre que la réputation de l'auditeur externe renforce le contenu informatif de la composante anormale du résultat publié. Cette composante anormale du résultat est, dans la lignée des travaux fondateurs de Jones (1991), mesurée en estimant les accruals anormaux, supposés discrétionnaires. L'argument central de Krishnan est que la qualité de l'audit, telle que perçue via la réputation de l'auditeur, permet de crédibiliser les accruals discrétionnaires inclus dans le résultat comptable. Or ce critère de réputation - s'il mérite d'être pris en compte - constitue une mesure relativement bruitée de la qualité effective du processus d'audit. D'autres critères sont venus récemment enrichir les travaux empiriques, qu'ils soient propres la relation auditeur-audité (par exemple la durée de la collaboration entre l'auditeur et son client), ou qu'ils relèvent de l'implication du conseil d'administration dans les problématiques d'audit à travers la mise en place d'un comité d'audit plus ou moins indépendant. En particulier, à notre connaissance, aucune étude n'a analysé l'impact des comités d'audit sur le contenu informatif des accruals anormaux. Cependant, différents travaux d'essence anglo-saxonne montrent que 
la présence d'un comité d'audit, mais également son indépendance vis-à-vis des dirigeants, incitent ces derniers à être plus prudents dans leurs choix comptables et à publier de faibles accruals anormaux (Klein, 2002a ; Bédard et al., 2004).

Dans cet article, nous élargissons au contexte français la problématique du contenu informatif des accruals discrétionnaires en lien avec la qualité du processus d'audit. Une confrontation de ce contexte aux travaux anglo-saxons s'avère particulièrement intéressante étant donné les spécificités françaises. Le cadre comptable auquel se réfèrent nos auditeurs, axé sur la règle plutôt que sur les principes, accorde notamment, et pour des raisons principalement historiques, une plus grande importance à l'application du principe de prudence. Dans un tel cadre la qualité de la signature d'audit externe devrait avant tout traduire la fiabilité des chiffres comptables plutôt que leur pertinence, et au regard de l'exigence de régularité des états financiers attestée par les commissaires aux comptes. Piot et Janin (2007) montrent cependant qu'en France, le recours à un cabinet d'audit réputé n'influe pas significativement sur la propension des entreprises à manipuler leur résultat. Suite aux rapports Viénot (1995 et 1999) puis au rapport Bouton (2002), les comités d'audit se sont largement développés au sein des grandes sociétés cotées françaises. Cependant leur création reste un acte volontaire, et seules des recommandations ont été rédigées sur leurs rôles, missions et fonctionnements. C'est ainsi que Thierry Breton, ancien ministre des finances, a tenté de diminuer sa propre responsabilité dans l'affaire Rhodia, en arguant que les comités d'audit n'ont « aucune existence légale ». Néanmoins, la volonté affichée par les institutions françaises de développer les comités d'audit et leurs prérogatives invitent à s'interroger sur l'influence de ces mécanismes organisationnels de gouvernance sur la gestion des chiffres comptables. Piot et Janin (2007) ont ainsi observé qu'en présence d'un comité d'audit, les entreprises tendent à réduire leur gestion du résultat via les accruals anormaux. Dans ce contexte, le comité d'audit influerait avant tout sur la fiabilité des chiffres comptables plutôt que sur leur pertinence, et donc leur utilité informative. Notre démarche empirique vise alors à tester l'impact de divers attributs de qualité du processus d'audit, en lien avec l'auditeur externe ou le comité d'audit, sur le contenu informatif des accruals discrétionnaires en France. La section 1 de l'article développe les hypothèses de recherche. La section 2 présente les aspects méthodologiques. La section 3 commente les résultats empiriques. En conclusion, nous résumons les principaux apports de ce travail. 


\section{Cadre d'analyse et hypothèses de recherche}

\subsection{Cadre conceptuel de l'analyse}

Le résultat comptable est généralement considéré comme ayant un contenu informatif supérieur aux flux de trésorerie quant à la valeur des entreprises. Il permet en effet d'enregistrer par le biais des accruals, en complément des dépenses et des recettes de l'exercice, des transactions qui, bien que n'ayant pas encore fait l'objet d'encaissements ou de décaissements, correspondent déjà à une création ou destruction de valeur. De nombreuses études empiriques s'appuyant sur la méthodologie des études d'association entre les rentabilités boursières et les variables comptables ont validé ces propriétés des états financiers, telles celles de Dechow (1994) pour les États-unis ou de Janin (2002) pour la France. Cependant, les accruals permettent aux dirigeants d'exercer leur discrétion pour adapter les états financiers en fonction des circonstances et de certaines exigences, et les résultats empiriques tendent à confirmer que les entreprises " gèrent » les chiffres comptables qu'elles publient en adaptant le niveau des accruals (Healy et Wahlen, 1999 ; Dumontier, 2003).

Les manipulations de résultats via les accruals anormaux peuvent répondre à deux grands objectifs (Holthausen, 1990 ; Healy et Palepu, 1993). Certaines recherches, les plus anciennes, considèrent que la gestion des accruals est motivée par des buts opportunistes tels que satisfaire aux exigences contractuelles, réglementaires, politiques, ou managériales auxquelles est exposée la firme, conformément à la théorie politico-contractuelle de la comptabilité (Watts et Zimmerman, 1986, 1990). D’autres recherches, plus récentes, considèrent que les accruals sont manipulés par les entreprises dans le but de signaler aux investisseurs les réelles perspectives d'avenir, l'information comptable ne permettant pas à elle seule d'atteindre cet objectif à cause de la rigidité des principes qui gouvernent son élaboration (Subramanyam, 1996 ; Guay et al., 1996 ; Krishnan, 2003). Dans cette seconde perspective, axée sur la réduction des asymétries informationnelles entre la firme et ses partenaires extérieurs, la manière dont les chiffres comptables sont validés par le processus d'audit apparait comme une dimension importante du problème tant pour les producteurs que pour les utilisateurs de l'information financière. En d'autres termes, la qualité de la vérification des accruals importe à divers endroits, comme nous le verrons par la suite.

La qualité intrinsèque du travail d'audit n'est toutefois pas observable pour l'utilisateur de l'information financière. Ces derniers doivent donc fonder leur évaluation sur une qualité apparente, ou perçue, du processus d'audit dans son ensemble. Traditionnellement, cette perception s'appuie largement sur la condition de l'auditeur externe, 
en ligne avec la définition conceptuelle donnée par DeAngelo (1981) : la qualité de l'audit se définit alors comme la probabilité que l'auditeur externe détecte une anomalie présente dans les états financiers (sa compétence), et la révèle au marché (son indépendance). Toutefois, et notamment en France depuis le premier rapport Viénot (1995), la qualité du processus d'audit intègre de plus en plus le rôle de surveillance émanant des comités d'administrateurs, et en l'occurrence des comités d'audit. En conséquence, nous intégrons cet élargissement du concept de qualité de l'audit en développant nos hypothèses au niveau de l'auditeur externe tout d'abord, et du comité d'audit ensuite.

\subsection{Gestion du résultat, auditeur externe et signal}

Les auditeurs externes doivent notamment veiller au respect des deux qualités essentielles de l'information énoncées dans la plupart des cadres conceptuels, à savoir fiabilité et pertinence. Sous l'angle de la fiabilité, c'est-à-dire l'absence d'irrégularités significatives, l'auditeur est là en tant que rempart contre l'opportunisme managérial. Sous l'angle de la pertinence, c'est-à-dire l'utilité pour la prise de décisions économiques, l'auditeur est en quelques sortes un agent qui permet d'optimiser le signal véhiculé par la composante discrétionnaire du résultat comptable. Globalement, la qualité de l'auditeur externe devrait permettre aux utilisateurs de l'information comptable de distinguer entre ces deux alternatives. La capacité d'un auditeur à se montrer rigoureux et indépendant est généralement appréciée par sa taille (DeAngelo, 1981) ou par sa réputation (Klein et Leffler, 1981). Globalement, les grands cabinets d'audit (les Big N) ont la réputation de fournir des services d'audit de qualité différenciée; leur signature devrait donc avoir un effet stimulant sur la fiabilité et la pertinence des chiffres comptables.

Néanmoins, si l'on considère la dimension informative des chiffres comptables (le volet «pertinence »), les résultats empiriques actuels restent peu nombreux et non consensuels. Deux études de réactions américaines basées sur l'annonce des résultats comptables se contredisent. La première, conduite sur la décade 1980 par Teoh et Wong (1993), montre que les coefficients de réponse (Earning Response Coefficients, ERC) sont statistiquement inférieurs parmi les firmes non auditées par les Big N. La seconde, conduite sur la période 1990-2000 par Ghosh et Moon (2005) avec 38794 observations, montre au contraire que les ERC sont plus faibles pour les firmes auditées par les Big N. Krishnan (2003) s'appuie sur un modèle d'association entre rentabilité boursière et performance comptable, et décompose cette dernière entre flux de trésorerie opérationnel, accruals normaux et accruals anormaux. Son étude, sur la période 1989-1998, confirme que les 
accruals anormaux ont bien un contenu informatif, et que le fait d'être audité par un Big N accroît ce contenu informatif.

À la lumière de ces études américaines, notre argumentation se propose de « revisiter » l'approche de Krishnan sur le marché actions français, dans un contexte normatif sensiblement différent. La contribution « informative » des grands cabinets d'audit, mise en évidence outre-atlantique, est alors loin d'être systématique. En effet, les deux composantes de qualité des chiffres comptables sur lesquels agissent les grands cabinets sont en conflit : il est couramment admis qu'une recherche de fiabilité accrue se fait au détriment de la pertinence de l'information. Par exemple, les délais de vérification et de publication allongés du fait de contrôles étendus entraîneront une disponibilité plus tardive de l'information pour les investisseurs, avec pour résultat une utilité réduite de cette dernière en matière décisionnelle, et un risque accru de transactions «initiées ». D’une manière générale donc, l'objectif de conformité «absolue » a pour conséquence une perte de contenu informatif des données comptables diffusées sur le marché. Cette contradiction justifie, entre autres, le recours à des cadres comptables moins rigides, basés sur les principes plutôt que sur des règles strictes. Il en résulte, dans cette optique, une sollicitation croissante du jugement professionnel dont doivent faire preuve les auditeurs externes.

Le cadre comptable français - examiné dans la présente étude - se distingue de la plupart des cadres conceptuels anglo-saxons sur plusieurs points. Historiquement, il est tout d'abord orienté vers les prêteurs de capitaux (banques, institutions financières), avec une priorité forte donnée à l'application du principe de prudence. L'objectif est alors de garantir aux créanciers l'information relative à la solvabilité de la firme, plutôt que d'informer un actionnariat externe assez réduit sur les perspectives de performance future. Le cadre français est ensuite axé sur la règle (ruled-based accounting) plutôt que sur les principes: la conformité aux textes y occupe une place centrale, au nom du principe de régularité attesté, d'ailleurs, par les auditeurs. Transposé à la relation inverse entre fiabilité et pertinence, ce cadre comptable suggère alors qu'une application excessive du principe de prudence peut être nuisible à l'utilité informative des chiffres comptables. En replaçant la dimension informative des chiffres comptables dans le débat sur la théorie des signaux, il devient possible d'anticiper l'impact pénalisant, sur cette dimension, d'un excès de qualité « conformiste » de l'audit. Aux États-Unis, cadre conceptuel également axé sur la règle (Webster et Thornton, 2004), plusieurs études empiriques tendent à montrer que la présence d'un grand cabinet d'audit (un Big N) agît comme un rempart à la gestion du résultat comptable ${ }^{\mathrm{ii}}$. L'étude de Kim et al. (2003) montre notamment que les Big N se différencient des autres cabinets par leur attitude 
prudente vis-à-vis des choix comptables. Dès lors, en recherchant une conformité maximale dans leur démarche de qualité de l'audit, ces Big N ont tendance à «brider » l'opportunisme des dirigeants certes (Becker et al., 1998), mais aussi, par conséquent, la flexibilité comptable dont disposent ces derniers pour informer le marché sur les perspectives de rentabilité. En d'autres termes, dans un cadre comptable tourné vers la prudence comme c'est le cas en France, les Big $\mathrm{N}$ vont avoir tendance à réduire l'effet de signal associé à la composante discrétionnaire du résultat publié. D'où notre première hypothèse :

H1a : L'association entre les accruals discrétionnaires et les rentabilités boursières est moins forte pour les entreprises auditées par des Big $\mathrm{N}$ que pour les entreprises non auditées par des Big $\mathbf{N}$.

Il a souvent été reproché aux facteurs de réputation / taille de l'auditeur de mal représenter la qualité effective du service d'audit. L'actualité, avec plusieurs scandales financiers impliquant les grands noms de l'audit, s'est d'ailleurs largement faite l'écho de ces critiques. Le principal problème des variables catégorielles, généralement binaires, limitées à la réputation globale de l'auditeur réside dans son incapacité à tenir compte, au cas par cas, des spécificités de la relation auditeur-audité. Or, un cabinet peut avoir une bonne réputation générale de compétence et d'indépendance, et se trouver dans une position fragile en termes d'indépendance vis-à-vis d'un client donné. Par conséquent, diverses approches un peu plus « fines » de la qualité de l'audit sont venues enrichir ces dernières années le champ de recherche empirique. Parmi ces mesures, la durée de la relation auditeur-audité a été assez largement étudiée dans les environnements d'audit anglo-saxons.

Les normalisateurs et les autorités de marché avancent qu'une association trop longue entre l'auditeur et son client constitue une menace à la diligence, et surtout à l'indépendance du premier. L'étude expérimentale de Knapp (1991), sur des membres de comités d'audit américains, révèle que la capacité à détecter une anomalie s'accroît au cours des premières années du mandat d'audit, puis diminue graduellement pour atteindre son niveau le plus bas après 20 années de collaboration. Ainsi, les partisans de la rotation obligatoire des mandats d'audit estiment qu'une longue relation auditeur-audité traduit des liens de familiarité susceptibles à la fois d'entacher l'apparence d'indépendance, mais également de placer l'auditeur dans une situation de laxisme, avec pour effet une moindre vigilance vis-à-vis des sources potentielles de risques et d'irrégularités.

Toutefois, la théorie générale selon laquelle la qualité de l'audit diminue avec la durée de la relation auditeur-audité ne trouve, pour l'heure, aucun support empirique; elle tend 
même à être contredite. En termes de gestion du résultat, les travaux disponibles actuellement ne couvrent que des échantillons américains. Parmi eux, Lin et al. (2006) trouvent que la probabilité d'une correction d'erreur (rétroactive) n'est pas associée à l'ancienneté de l'auditeur. Frankel et al. (2002) observent une relation négative entre la durée de la relation auditeur-audité et les accruals anormaux en valeur absolue, ainsi qu'un effet modérateur de cette durée parmi les firmes qui gèrent leur résultat à la baisse. De manière similaire, Myers et al. (2003) constatent que l'ancienneté de l'auditeur se traduit par une réduction des accruals anormaux à la fois positifs et négatifs. Les travaux plus récents de Menon et Williams (2004), et de Reynolds et al. (2004) confirment l'association négative entre la durée de la relation auditeur-audité en années, et les accruals anormaux en valeur absolue.

Johnson et al. (2002) catégorisent la durée de la relation auditeur-audité comme courte (2-3 ans), moyenne (4-8 ans), ou longue (9 ans ou plus). En étudiant les firmes auditées par les Big N sur la période 1986-1995, ils observent qu'une relation courte est associée à une réduction des accruals anormaux, et qu'une relation longue reste sans effet sur cette mesure de gestion du résultat. Nagy (2005) reprend la même codification et s'intéresse à la période 2000-2003, avant et après la faillite d'Andersen. Il constate que les relations courtes et longues sont toutes deux associées positivement à l'ampleur des accruals anormaux, suggérant ainsi l'existence d'une durée optimale de la relation auditor-audité en matière de gestion du résultat. Enfin, en termes de contenu informatif, l'unique étude réalisée provient de Ghosh et Moon (2005), et fait état de coefficients de réponse (ERC) plus forts lorsque la durée de relation auditeur-audité s'accroît, et ce à partir d'un large échantillon de firmes américaines sur la décade 1990-2000.

En résumé, la plupart des travaux empiriques disponibles réfutent l'hypothèse d'une érosion de la qualité de l'audit avec le temps. Bon nombre d'entre elles corroborent même le contraire, suggérant ainsi que l'auditeur externe gagne en compétence au fil des années en profitant d'une meilleure connaissance ou expérience de la firme auditée. Cette interprétation ad hoc mérite toutefois d'être documentée empiriquement. Plus généralement, il peut être avancé que la forte contradiction entre postulats théoriques et constatations empiriques vient d'un puzzle, lié au fait que la durée de la collaboration auditeur-audité a des effets contraires sur les composantes de qualité de l'audit, comme le montre la figure 1. 
Figure 1 : Durée de la relation auditeur-audité, qualité de l'audit et contenu informatif de la gestion du résultat

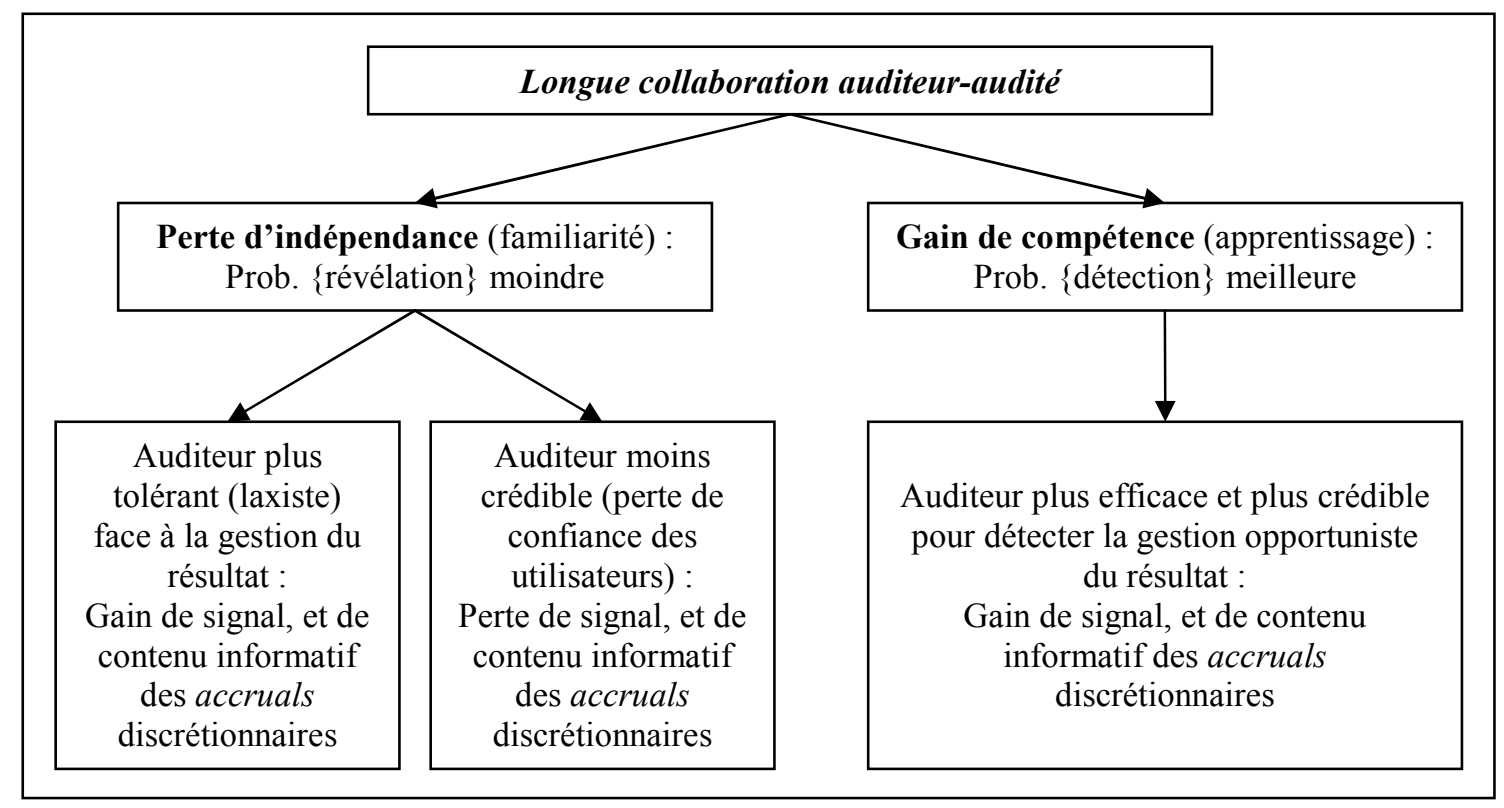

Aussi, devant la complexité du phénomène, et l'ambiguïté qu'il déclenche, nous posons notre seconde hypothèse de recherche sous sa forme nulle :

H1b : L'association entre les accruals discrétionnaires et les rentabilités boursières n'est pas altérée par la durée de la relation auditeur-audité.

\subsection{Gestion du résultat, comité d'audit et signal}

Avec un temps de retard, les principes de gouvernance anglo-saxons visant à renforcer l'implication du conseil d'administration dans la surveillance managériale prennent une dimension institutionnelle en France suite au premier rapport Viénot (1995). C'est notamment ce document qui agit comme véritable catalyseur de la mise en place volontaire de comités d'audit, chargés de valider les principales options comptables retenues par les dirigeants, de rencontrer en privé les auditeurs (internes et externes) afin de maximiser la probabilité de révélation d'irrégularités, et de renforcer l'indépendance de l'auditeur externe en intervenant dans les phases de sélection-renouvellement-congédiement du cabinet d'audit, et de négociation de ses honoraires. Dans une optique de surveillance effective des choix comptables, l'efficacité du comité d'audit suppose, à tout le moins, un certain degré d'indépendance par rapport aux dirigeants.

Les travaux empiriques qui considèrent l'effet de la présence d'un comité d'audit, ou de son indépendance, sur le contenu informatif des chiffres comptables sont là encore 
essentiellement américains, et peu nombreux. En termes de simple présence, Wild (1994, 1996) considère 260 firmes cotées ayant volontairement (à l'époque) formé un comité d'audit sur la période 1966-1980. Il observe que ces firmes ont significativement accru le contenu informatif de leurs résultats l'année qui suit la mise en place du comité. Il base cette conclusion sur l'observation comparative avant / après comité d'audit de deux indicateurs : le coefficient de réponse à l'annonce du résultat (ERC), et la variance anormale des rendements journaliers autours de la date d'annonce. En termes d'indépendance du comité d'audit, les résultats restent encore disparates. Anderson et al. (2003) examinent les grandes firmes américaines pour l'année 2000 (1241 observations) ; leur modèle montre que la proportion d'administrateurs indépendants dans le comité d'audit n'améliore pas le contenu informatif du résultat non anticipé (l'ERC). En revanche, Bryan et al. (2004), à partir des firmes du S\&P500 sur la période 1996-2000 (1835 observations), constatent que le fait de disposer d'un comité d'audit 100\% indépendant donne à la firme un ERC significativement supérieur, donc un contenu informatif accru lors de l'annonce de son résultat comptable annuel. Un tel résultat n'est toutefois pas confirmé par Petra (2007) au niveau des annonces de résultats trimestriels, bien que son étude couvre une population relativement similaire à celle de Bryan et al. (765 observations relatives aux firmes du S\&P500 sur la période 1996-1999).

Globalement, ces résultats non consensuels, voire contradictoires, sont possiblement attribuables à une argumentation théorique incomplète quant à l'effet d'une surveillance accrue du processus d'audit sur le contenu informatif des chiffres comptables. Notons également que les quelques travaux empiriques disponibles n'isolent pas la composante discrétionnaire du résultat comptable, et considèrent la fraction non anticipée de ce dernier par le marché. Ainsi, à l'instar des développements proposés plus haut pour les grands cabinets d'audit, il se peut qu'en ajoutant une contrainte à la flexibilité comptable, les comités d'audit trop conformistes finissent par «brider » l'effet de signal induit par la gestion du résultat, entraînant alors une perte de continu informatif.

En France, le principe de volontariat n'a pas été remis en cause concernant la formation même d'un comité d'audit, contrairement aux exigences boursières américaines. Néanmoins, les recommandations minimales d'indépendance, absentes du rapport Viénot I, ont été renforcées par les groupes de travail subséquents. Ainsi, le seuil recommandé est passé de un tiers (seulement!) d'administrateurs indépendants dans le rapport Viénot II (1999), à deux tiers dans le rapport Bouton (2002). Dès lors, si le phénomène des comités d'audit marque, probablement, un certain mimétisme des pratiques anglo-saxonnes, la contrainte de surveillance (monitoring) semble belle et bien présente au niveau de la demande de tels 
mécanismes organisationnels de gouvernance. À ce titre, Piot (2004) constate, deux ans après le rapport Viénot I, que les problèmes d'agence et d'asymétrie informationnelle, notamment l'ampleur des conflits actionnaires-dirigeants, ressortent parmi les principaux déterminants de la présence d'un comité d'audit indépendant dans les firmes françaises cotées. Plus récemment, Piot et Janin (2007) trouvent que les firmes françaises dotées d'un comité d'audit ont des accruals discrétionnaires moins élevés que les autres. Cela suggère que la présence d'un comité d'audit, qui plus est indépendant, est de nature à contribuer à la qualité d'ensemble du processus d'audit en renforçant la contrainte, autrement dit le «bridage » des politiques comptables. Par conséquent, en ligne avec la première hypothèse sur la réputation de l'auditeur externe (H1a), nous émettons deux hypothèses, relatives à la présence d'un comité d'audit tout d'abord, puis à son indépendance. Globalement, ces deux hypothèses suggèrent que la présence d'un comité d'audit, et l'indépendance de ce dernier, sont de nature à réduire la flexibilité comptable, donc le contenu informatif des accruals discrétionnaires.

H2a : L'association entre les accruals discrétionnaires et les rentabilités boursières est moins forte pour les entreprises qui disposent d'un comité d'audit.

H2b : L'association entre les accruals discrétionnaires et les rentabilités boursières est moins forte pour les entreprises qui disposent d'un comité d'audit indépendant.

\section{Aspects méthodologiques}

\subsection{L'échantillon d'entreprises}

Les entreprises visées sont celles incluses dans l'indice SBF 120 sur la période 19992001. Pour donner à l'analyse une dimension longitudinale et éviter les limites habituelles d'une simple étude en coupe transversale, nous travaillons sur trois années consécutives. Nous nous concentrons sur ces exercices car cela nous permet de couvrir la période comprise entre la publication du second rapport Viénot (juillet 1999) et celle du rapport Bouton (septembre 2002). Cette période marque une première phase de maturité dans la diffusion des comités d'audit au sein des sociétés cotées françaises (Piot, 2004). En outre, elle fait référence à un contexte d'audit relativement serein, car non encore bouleversé par les turbulences du scandale Enron-Andersen.

L'échantillon de travail est composé de 102 firmes. Il correspond globalement aux sociétés qui sont entrées dans l'indice SBF 120 au moins une fois sur la période 1998-2002, et qui n'exercent pas une activité financière ou assimilée ${ }^{\mathrm{iii}}$. Le tableau 1 donne des précisions sur les différentes étapes de la procédure d'obtention de l'échantillon. Les variables comptables 
nécessaires à notre étude sont issues de la base Diane, les données boursières ajustées de la base Datastream, et les données d'audit des rapports annuels. Les informations manquantes dans les différentes bases, ainsi que les contraintes relatives à l'estimation des accruals discrétionnaires (voir ci-après), nous ont amené à supprimer 32 entreprises pour 1999, 27 entreprises pour 2000 et 21 entreprises pour 2001. De plus, nous avons éliminé les observations dont les rentabilités boursières et le résultat net étaient soit inférieurs au centile 2,5\%, soit supérieurs au centile $97,5 \%{ }^{\text {iv }}$. L'échantillon final sur lequel ont été testés les modèles est composé de 152 entreprises-année, formé par 67 entreprises différentes.

[Insérer tableau 1]

\subsection{Le calcul des accruals discrétionnaires}

Les engagements (Total Accruals, TA) tels que nous les calculons correspondent, sur la base de données comptables consolidées, à la différence entre le résultat net de l'entreprise $(\mathrm{RN})$ et le flux de trésorerie opérationnel (Cash from operations, CFO) :

$$
\mathrm{TA}=\mathrm{RN}-\mathrm{CFO} \Leftrightarrow \mathrm{RN}=\mathrm{CFO}+\mathrm{TA}
$$

Nous avons calculé les accruals totaux (TA) pour chaque firme i et année t selon la formule suivante :

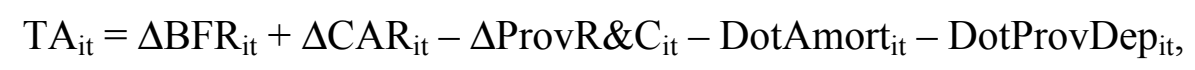

avec $\mathrm{i}=1$ à $102, \mathrm{t}=1999,2000,2001$, et :

- $\quad \Delta \mathrm{BFR}=$ variation du besoin en fonds de roulement calculée avec les valeurs brutes d'actif circulant. BFR $=($ Stock + Clients + Autres créances + Ch. d'avance $)-($ Fournisseurs + Dettes fiscales \& sociales + Autres dettes + Pdts d'avance)

- $\quad \Delta \mathrm{CAR}=$ variation des Charges à Répartir à l'actif du bilan ;

- $\triangle$ ProvR\&C = variation des Provisions pour Risques et Charges au passif du bilan ;

- DotAmort $=$ Dotation aux Amortissements d'exploitation au compte de résultat ;

- DotProvDep = Dotation aux Provisions pour Dépréciation des actifs circulants, obtenue par variation de la différence (Actif Circulant Brut - Actif Circulant Net).

Dans un deuxième temps, nous estimons les accruals discrétionnaires, c'est-à-dire susceptibles de résulter de manipulations comptables. À l'instar de nombreuses études sur cette question, la composante discrétionnaire est estimée à l'aide de la version modifiée (Dechow et al., 1995) du modèle transversal intra-sectoriel de Jones (1991) en retenant les secteurs d'activité sur la base du code NAF à trois chiffres et d'un nombre minimum de six sociétés par secteur ${ }^{\mathrm{v}}$.

Formellement, nous avons donc estimé le modèle suivant pour chaque secteur d'activité $\mathrm{j}$ et année $\mathrm{t}$ :

$$
\mathrm{TA}_{\mathrm{jt}}=\alpha 1+\beta 1 . \mathrm{IMMOS}_{\mathrm{jt}}+\gamma 1 . \Delta(\mathrm{CA}-\mathrm{CLIENTS})_{\mathrm{jt}}+\varepsilon_{\mathrm{jt}},
$$


avec (toutes ces variables y compris la constante $\alpha$ sont normées par le total de l'actif en début d'exercice) :

- $\quad \mathrm{TA}=$ Total Accruals ;

- $\quad$ IMMOS = Immobilisations corporelles brutes + charges à répartir ;

- $\mathrm{CA}=$ Chiffre d'affaires ;

- CLIENTS = Créances clients en valeur brute.

Les accruals discrétionnaires (discretionary accruals, DAC) sont enfin obtenus par différence entre les accruals totaux de chaque firme et les accruals supposés non discrétionnaires déterminés à l'aide des paramètres du modèle modifié de Jones. Formellement, pour chaque firme i et année t, on obtient alors :

$$
\mathrm{DAC}_{\mathrm{it}}=\mathrm{TA}_{\mathrm{it}}-\left[\alpha 1+\beta 1 . \mathrm{IMMOS}_{\mathrm{it}}+\gamma 1 . \Delta(\mathrm{CA}-\mathrm{CLIENTS})_{\mathrm{it}}\right],
$$

Le tableau 2 donne des statistiques descriptives globales sur les diverses variables d'accruals, totaux et discrétionnaires.

\section{[Insérer tableau 2]}

Les accruals discrétionnaires sont en moyenne positifs et significativement différents de zéro. En outre, une ANOVA unidimensionnelle sur les trois années ne révèle pas de différences significatives.

\subsection{Le modèle d'association}

A l'instar de Subramanyam (1996) et Krishnan (2003), nous apprécions le contenu informatif des accruals discrétionnaires en décomposant le résultat en trois éléments - les flux de trésorerie opérationnel, les accruals discrétionnaires et les accruals non discrétionnaires - et en testant leur association avec les rentabilités boursières :

$$
\begin{aligned}
\mathrm{RETt}= & \beta 0+\beta 1 \mathrm{CFOt}+\beta 2 \mathrm{DACt}+\beta 3 \mathrm{NDACt}+\beta 4 \mathrm{DACt} * \mathrm{Xt}+\beta 5 \mathrm{Xt}+\beta 6 \mathrm{Y} 1999+\beta 7 \\
& \mathrm{Y} 2000+\varepsilon
\end{aligned}
$$

avec :

- RET les rentabilités boursières calculées sur une période de 12 mois se terminant 4 mois après la date de clôture des comptes ;

- CFO : le flux de trésorerie opérationnel (résultat net - accruals totaux) par action ;

- DAC : les accruals discrétionnaires par action;

- $\mathrm{NDAC}^{\mathrm{vi}}$ : les accruals non discrétionnaires par action (TA - DAC) ;

- Y1999 (Y2000) : variable binaire de contrôle des effets annuels, codée 1 si l'observation est issue de l'année 1999 (2000), et 0 sinon.

X représente les différentes variables d'audit successivement testées :

- BIG5 : variable binaire codée 1 si au moins un des commissaires aux comptes titulaires fait partie des Big Five, et 0 sinon;

- Tenure : variable binaire codée 1 si le nombre d'années de fonction du commissaires aux comptes dominant est supérieur à 6 et 0 sinon $^{\text {vii }}$; 
- $\mathrm{AC}$ : variable binaire codée 1 si la société a mis en place un comité d'audit et 0 sinon ;

- ACIND : variable binaire codée 1 si la société a mis en place un comité d'audit composé de plus de $50 \%$ de membres indépendants au sens large, à savoir des administrateurs non exécutifs, ne faisant pas partie des actionnaires de référence, et sans liens apparents avec des membres de la haute direction ou des actionnaires de référence.

Les coefficients $\beta 1, \beta 2$ et $\beta 3$ sont attendus positifs. Aucune prédiction n'est proposée pour le coefficient $\beta 5$. Le coefficient $\beta 2$ traduit l'association entre les accruals discrétionnaires et les rentabilités boursières indépendamment de la variable d'audit testée. La somme de $\beta 2$ et de $\beta 4$ mesure l'intensité de cette même association, lorsque sont prises en compte les variables d'audit. Pour la variable BIG5, dans la mesure où les cabinets les plus réputés vont avoir tendance à réduire l'effet de signal associé à la composante discrétionnaire du résultat publié (H1a), $\beta 4$ est attendu négatif. La durée du mandat des commissaires aux comptes pouvant tout à la fois, et de façon contradictoire, être un facteur d'aliénation aux visées des dirigeants et un gage d'expertise renforcée des auditeurs (H1b), nous n'avons pas posé d'hypothèse sur le signe de la variable Tenure. Les comités d'audit, qui plus est lorsqu'ils sont indépendants, ayant pour fonction de contrôler la politique comptable des entreprises, leur présence devrait atténuer l'effet de signal associé aux accruals discrétionnaires. $\beta 4$ est donc attendu négatif pour les variables AC et ACIND (H2a et H2b).

\section{Résultats empiriques}

\subsection{L'analyse des statistiques univariées}

Le tableau 3 reporte les statistiques descriptives sur les différentes composantes du résultat et sur les accruals anormaux, selon les caractéristiques du processus d'audit externe.

\section{[Insérer tableau 3]}

Les sociétés audités par un cabinet réputé (Big $\mathrm{N}$ ) présentent en moyenne et en médiane un résultat net significativement inférieur au résultat net publié par les sociétés non auditées par un Big N. Par contre, le flux de trésorerie opérationnel (CFO), qui correspond par construction à la composante non manipulable du résultat, n'est pas significativement différent entre les deux sous-échantillons. L'écart de performance comptable entre les deux sous-échantillons s'explique donc par une composition différente des résultats comptables : les accruals totaux des sociétés auditées par un Big $\mathrm{N}$ sont négatifs ( $\mathrm{t}$ de student de -3,005 pour l'hypothèse de nullité, non reporté dans le papier) et significativement inférieurs aux accruals totaux des sociétés non auditées par un Big N. Ceci est cohérent avec les résultats de Francis et al. (1999), qui montrent que les sociétés qui publient des accruals élevés ont une 
plus forte probabilité de nommer un grand cabinet (Big 6 dans leur étude), dans le but de crédibiliser les chiffres comptables livrés aux investisseurs. Il faut cependant préciser que pour les sociétés non auditées par un Big N, la médiane des accruals totaux est négative, et non significativement différente de la médiane des sociétés auditées par un Big N, alors que la moyenne, elle, est légèrement positive. Cette moyenne est donc influencée à la hausse par quelques sociétés publiant des accruals « excessivement positifs ».

Les sociétés auditées par un Big $\mathrm{N}$ publient des accruals discrétionnaires signés (DAC) légèrement positifs, mais significativement inférieurs aux accruals discrétionnaires des autres sociétés $(+0,008$ contre $+0,057$ en moyenne). En médiane, ces écarts ne sont cependant pas statistiquement significatifs. Abstraction faite du sens de la gestion du résultat (à la hausse ou à la baisse), il apparaît que les sociétés non auditées par un Big N présentent des accruals discrétionnaires en valeur absolue (ABS DAC) significativement plus élevés que les sociétés auditées par un Big N. Ces statistiques suggèrent donc que les sociétés auditées par un «grand cabinet» sont globalement plus prudentes que les autres dans leur publication d'accruals discrétionnaires. Les résultats de Francis et al. (1999) ou de Krishnan (2003), dans le contexte américain, suggèrent également que les grands cabinets sont plus conservateurs dans leurs choix comptables que leurs concurrents de moindre réputation. Or, selon nous, si cette propension à la prudence comptable permet bien de garantir les utilisateurs contre d'éventuelles manipulations opportunistes des comptes, elles peuvent aussi, à l'opposé, nuire à la qualité du signal émis via une gestion adéquate du résultat. Une trop forte tendance à l'orthodoxie comptable ne permet pas d'envoyer des signaux suffisamment explicites pour qu'ils ne soient pas ambigus et peut nuire, par là même, à l'intelligibilité de ces signaux.

La durée du mandat de l'auditeur externe ne semble pas influencer significativement la propension des entreprises à gérer leurs résultats comptables. En moyenne, parmi toute les variables comptables testées, seul le flux de trésorerie opérationnel, qui constitue en l'occurrence la seule donnée objective, est significativement différent entre les deux sous échantillons. Ces statistiques étaient attendues compte tenu des effets contradictoires que la durée de la relation auditeur-audité peut avoir sur la qualité de l'audit et donc de l'information comptable. Il est ainsi difficile d'arbitrer entre la perte d'indépendance que peut entraîner une durée trop longue de la collaboration entre l'auditeur et son client, postulat théorique le plus courant dans la littérature, et le gain de compétence associé à une relation durable que met en évidence la plupart des travaux empiriques (cf. figure 1). 
Le tableau 4 reporte les statistiques descriptives sur les différentes composantes du résultat et sur les accruals anormaux, selon la présence et les caractéristiques d'indépendance des comités d'audit.

\section{[Insérer tableau 4]}

Nous avons tout d'abord comparé les sociétés avec comité d'audit (sous échantillon A) à l'ensemble des autres sociétés (sous échantillon D). Les sociétés ayant un comité d'audit présentent un résultat comptable en moyenne moins élevé que les autres sociétés, alors même que les flux de trésorerie opérationnels ne sont pas statistiquement différents entre les deux populations. La plus faible performance comptable des sociétés avec comité d'audit s'explique donc, comme pour les sociétés auditées par un Big N, par le fait qu'elles présentent des accruals totaux négatifs et d'amplitude plus importante que les autres sociétés. Les sociétés avec comité d'audit publient des accruals discrétionnaires signés (DAC) légèrement négatifs mais significativement inférieurs à ceux des autres sociétés $(-0,005$ contre $+0,062$ en moyenne). On retrouve un écart de même sens et également statistiquement significatif pour les accruals discrétionnaires en valeur absolue (ABS DAC). Ceci est cohérent avec les résultats de Piot et Janin (2007) qui montrent que la présence d'un comité d'audit semble prévenir les comportements discrétionnaires des entreprises et plus particulièrement la gestion des accruals visant à augmenter le résultat.

Nous avons ensuite comparé les sociétés ayant un comité d'audit indépendant (sous échantillon B) respectivement aux sociétés sans comité d'audit (sous échantillon D), aux sociétés avec comité d'audit non indépendant (sous échantillon E) et enfin à l'ensemble de autres sociétés $(\mathrm{D}+\mathrm{E})$. On constate des écarts véritablement significatifs uniquement pour la comparaison avec les sociétés sans comité d'audit. Plus précisément, il apparaît que les sociétés sans comité d'audit publient des résultats plus élevés que ceux publiés par les sociétés avec comité d'audit indépendant $(+0,074$ contre $+0,045$ pour les moyennes), notamment du fait d'accruals discrétionnaires très largement positifs et significativement supérieurs aux accruals discrétionnaires des sociétés à comité d'audit indépendant $(+0,062$ contre $+0,008$ ). Indépendamment du sens de la gestion de leurs résultats (haussière ou baissière), les sociétés sans comité d'audit présentent également des accruals discrétionnaires significativement plus élevés que ceux des sociétés à comité d'audit indépendant $(0,101$ contre 0,061). In fine, si la simple nomination d'un comité d'audit ne semble pas devoir influencer les pratiques comptables des entreprises, sa composition apparaît, dans certains cas, comme un facteur discriminant. Plus précisément, les entreprises dont les comités d'audit sont indépendants sont globalement plus prudentes que les entreprises sans comité d'audit dans 
leur publication d'accruals discrétionnaires. Le risque est alors, selon nous, qu'à trop privilégier la fiabilité des chiffres comptables, les comités d'audit, dans la lignée des cabinets d'audit les plus réputés, atténuent les effets de signaux associés à une gestion adéquate des accruals.

\subsection{Le contenu informatif des accruals discrétionnaires}

Dans le tableau 5, nous présentons les résultats des différentes régressions selon les variables de qualité de l'audit que nous avons retenues : successivement la réputation du cabinet d'audit (BIG5), la durée du mandat du cabinet d'audit (Tenure), la présence d'un comité d'audit (AC) et l'indépendance du comité d'audit (ACIND).

\section{[Insérer tableau 5]}

Indépendamment $\mathrm{du}$ processus $\mathrm{d}$ 'audit, les trois variables composant le résultat comptable ont le signe attendu. Plus particulièrement, $\beta 2$ est positif et significatif au seuil de $1 \%$, ce qui induit que les accruals discrétionnaires véhiculent une information spécifique et utile au marché pour valoriser les sociétés. Les accruals discrétionnaires positifs (négatifs) sont perçus par le marché comme une bonne (mauvaise) nouvelle sur les profits futurs de la firme et ce au même titre que les accruals non discrétionnaires ( $\beta 3$ est également positif et significatif au seuil de $1 \%$ ).

Pour ce qui est des caractéristiques de l'auditeur externe, $\beta 4$ est significatif uniquement pour la variable BIG5 (au seuil de 5\%) et son signe est négatif, conformément à notre hypothèse 1a. La somme de $\beta 2$ et $\beta 4$ mesurant alors l'intensité du contenu informatif des accruals discrétionnaires pour les sociétés auditées par des Big 5, il apparaît que les accruals discrétionnaires publiés par ces dernières possèdent un contenu informatif plus faible pour le marché. Or, ces mêmes sociétés publient en moyenne des accruals discrétionnaires moins élevés, en valeurs algébriques et absolues, que les sociétés non audités par un Big 5 (voir le tableau 3). Ces résultats suggèrent premièrement que, conformément aux travaux précurseurs de Subramanyan (1996), les accruals discrétionnaires sont prix en compte par les investisseurs pour estimer la valeur d'une société. Deuxièmement, et conformément à notre hypothèse $1 \mathrm{a}$, ils montrent que lorsqu'une société est auditée par un cabinet de renom, le signal qu'elle peut émettre en manipulant ses accruals est moins apprécié par le marché qu'un même signal émis par une société auditée par un cabinet de moindre envergure. Ce dernier point est contraire aux résultats de Krishnan (2003) qui, aux États-Unis, met en évidence que l'association des accruals discrétionnaires avec les rentabilités boursières est plus forte pour les sociétés auditées par un grand cabinet. Il en conclut que le recours à un auditeur plus 
réputé permet de renforcer la crédibilité du résultat publié en minimisant le bruit que les accruals discrétionnaires peuvent induire. Il est certes possible que les grands cabinets d'audit possèdent l'expertise et le pouvoir nécessaire pour détecter et résister aux éventuelles velléités de leurs clients de procéder à des manipulations comptables opportunistes. Mais nous pensons également qu'à être trop conservateurs, ils peuvent diminuer la capacité des comptables à faire des choix, et donc à exprimer une opinion éclairée sur la santé financière de leur société. Dans cette optique, le cadre comptable français deviendrait alors le «catalyseur » de cette perte de signal.

Le fait que la durée du mandant de l'auditeur ne semble pas influencer significativement le contenu informatif des accruals discrétionnaires, et donc la pertinence du signal véhiculé aux investisseurs par ce biais, est finalement en cohérence avec une littérature académique empreinte de contradictions entre les hypothèses théoriques et les principaux résultats empiriques (cf. figure 1). Ainsi est-il probable que, dans notre échantillon, les effets induits par la perte d'indépendance et ceux induits par l'expertise accrue des auditeurs externes se neutralisent et expliquent, pour le moins, la faible significativité de la variable Tenure.

Pour ce qui est du contrôle exercé par les administrateurs sur les chiffres comptables, la simple présence d'un comité d'audit ne semble devoir influencer qu'à la marge le contenu informatif des accruals discrétionnaires. $\mathrm{La}$ variable $\mathrm{AC} * \mathrm{DAC}$ est bien négative, conformément au signe attendu, mais le coefficient $\beta 4$ n'est pas significatif à un seuil critique communément admis (la probabilité critique unilatérale, non reportée dans le tableau, est de 13,5\%). Il apparaît cependant que lorsque les comités d'audit sont indépendants, leur présence semble devoir influencer un peu plus à la baisse le contenu informatif des accruals discrétionnaires. Le coefficient $\beta 4$ est en effet négatif, conformément au signe attendu, et significatif au seuil de 10\% pour la variable ACIND*DAC. Or, nous avons également montré que les comités d'audit tendent, à l'instar des cabinets d'audit les plus réputés, à modérer les comportements discrétionnaires des entreprises, particulièrement en matière de gestion à la hausse du résultat. C'est pourquoi nous arguons à nouveau qu'un excès de conformisme comptable tel que celui affiché par les comités d'audit peut nuire à la pertinence des chiffres comptables. En effet, en contrariant à l'excès la souplesse dont bénéficient les entreprises dans l'application des règles comptables, les comités d'audit diminueraient leur capacité à véhiculer aux investisseurs, par une gestion adéquate des accruals, de l'information utile sur la valeur créée. 
Notons par ailleurs que $89 \%$ des sociétés de notre échantillon ayant un comité d'audit sont également auditées par un Big 5 (statistique non reportée dans les tableaux). Pour différencier l'influence respective de ces deux facteurs sur le contenu informatif des accruals discrétionnaires, nous avons testé le modèle général suivant où les variables $\mathrm{AC}$ et BIG5 sont introduites simultanément (les résultats de la régression sont reportés dans le tableau 6) :

$$
\begin{aligned}
\mathrm{RETt}= & \beta 0+\beta 1 \mathrm{CFOt}+\beta 2 \mathrm{DACt}+\beta 3 \mathrm{NDACt}+\beta 4 \mathrm{BIG} 5 \mathrm{t}^{*} \mathrm{DACt}+\beta 5 \mathrm{BIG} 5 \mathrm{t} \\
& +\beta 6 \mathrm{ACINDt} * \mathrm{DACt}+\beta 7 \mathrm{ACINDt}+\beta 8 \mathrm{Y} 1999+\beta 9 \mathrm{Y} 2000+\varepsilon
\end{aligned}
$$

\section{[Insérer tableau 6]}

Le coefficient $\beta 4$ est négatif et significatif au seuil de $5 \%$, témoignant à nouveau de l'influence des grands cabinets d'audit sur le contenu informatif des accruals discrétionnaires. En revanche, le coefficient $\beta 6$ est bien négatif, donc conforme au signe attendu, mais il n'est pas statistiquement significatif. Autrement dit, lorsqu'une société est par ailleurs auditée par un cabinet réputé, les comités d'audit indépendants n'influencent pas spécifiquement le contenu informatif des accruals discrétionnaires. In fine, si la réputation des cabinets d'audit semble réellement influencer la politique comptable et l'utilité informative du résultat comptable des entreprises, l'efficacité des comités d'audit, structures émanant directement des conseils d'administration, n'apparaît pas probante. Ce résultat nous amène donc à nous interroger sur l'efficacité réelle, et notamment la compétence, de ces mécanismes de gouvernance.

\section{Conclusion}

Dans cette étude, nous abordons la question du contenu informatif des chiffres comptables publiés par les grandes firmes françaises, et plus particulièrement l'impact de divers attributs de qualité du processus d'audit financier sur ce contenu informatif : réputation et ancienneté de l'auditeur externe, et contrôle exercé par un comité d'audit indépendant. Le principal contraste avec l'environnement anglo-saxon réside dans un cadre comptable normatif fortement tourné vers la prudence, visant avant tout à évaluer la solvabilité de la firme plutôt que sa performance. Dès lors, la régularité et la sincérité des comptes, attestée par les auditeurs externes, privilégie souvent la conformité juridique des transactions plutôt que leur substance économique. Il en ressort que la qualité de la signature d'audit, dans un tel cadre, devrait plutôt traduire la fiabilité que la pertinence des chiffres comptables. Ainsi, nous anticipons que le contenu informatif de la composante discrétionnaire du résultat comptable est moindre, globalement, lorsque ce résultat est certifié par un grand cabinet d'audit (Big N), 
lorsque la firme s'est dotée d'un comité d'audit, et que ce comité d'audit comprend une majorité d'administrateurs indépendants. En revanche, au vu d'effets contradictoires sur la qualité de l'audit d'une (trop ?) longue collaboration entre l'auditeur et son client, nous émettons une hypothèse non directionnelle quant à l'impact de la durée de la relation auditeur-audité sur le contenu informatif des chiffres comptables.

Nos résultats empiriques, basés sur la méthodologie des études d'associations, confirment que, globalement, le contenu informatif des régularisations comptables (accruals) estimées comme étant discrétionnaires est moindre en présence d'un auditeur appartenant aux Big N, ou en présence d'un comité d'audit en majorité indépendant de la direction de la firme. La simple présence d'un comité d'audit, peu importe son degré d'indépendance, n'altère pas significativement le contenu informatif des accruals discrétionnaires; une interprétation de cela est que les comités qui n'ont pas un minimum d'indépendance ne parviennent pas à «brider» la flexibilité comptable des dirigeants, et par voie de conséquence la dimension informative des chiffres comptables. Enfin, une «longue» collaboration auditeur-audité, matérialisée dans notre étude par le dépassement du seuil de six années, reste également sans effet significatif sur l'association entre rentabilité boursière et composante discrétionnaire de la performance comptable.

Nos résultats - issus du cadre comptable français - contribuent à éclairer le débat actuel, dans le cadre notamment de l'adoption des normes IAS/IFRS, sur la contradiction apparente entre la fiabilité et la pertinence des chiffres comptables. Pour le moins, il semblerait qu'une application [excessive ?] du principe de prudence soit nuisible à l'utilité informative des chiffres comptables. Dans leur ensemble, nos conclusions militent donc en faveur du référentiel IFRS, dont le cadre conceptuel, basé sur des principes plutôt que sur des règles prudentielles, devrait permettre aux auditeurs de renom et aux organes de surveillance de mieux exercer leur jugement professionnel dans le sens de la pertinence des états financiers. Mais cette «priorité » donnée aux investisseurs en fonds propres conviendra-t-elle aux créanciers de la firme? 


\section{Bibliographie}

Anderson K. L., Deli D. N., Gillan S. L. (2003) «Boards of Directors, Audit Committees and the Information Content of Earnings », Working paper No. 2003-04, Weinberg Center for Corporate Governance, University of Delaware, http://ssrn.com/abstract=444241.

Becker C., DeFond M., Jiambalvo J., Subramanyam K. R. (1998) «The Effect of Audit Quality on Earnings Management », Contemporary Accounting Research $15: 1-24$.

Bouton D. (2002) « Pour un meilleur gouvernement des entreprises cotées - Rapport du groupe de travail présidé par Daniel Bouton », Document Mouvement des Entreprises de France (MEDEF) \& Association Française des Entreprises Privées (AFEP-AGREF), Paris.

Brown Jr W. D., He H., Teitel K. (2006) «Conditional Conservatism and the Value Relevance of Accounting Earnings: An International Study », European Accounting Review 15 : 605-626.

Bryan D., Liu M. H. C., Tiras S. L. (2004) « The Influence of Independent and Effective Audit Committees on Earnings Quality », Working paper, http://ssrn.com/abstract=488082.

Butler M., Leone A. J., Willenborg M. (2004) « An Empirical Analysis of Auditor Reporting and its Association with Abnormal Accruals », Journal of Accounting and Economics $37: 139-165$.

Christie A. (1987) « On Cross-Sectional Analysis in Accounting Research », Journal of Accounting and Economics $9: 231-258$.

DeAngelo L. E. (1981) «Auditor Size and Audit Quality », Journal of Accounting and Economics 3(2) : 183199.

Dechow P. M. (1994) «Accounting Earnings and Cash Flows as Measures of Firm Performance: The Role of Accounting Accruals », Journal of Accounting and Economics 18 : 3-42.

Dechow R. M., Sloan R. G., Sweeney A. P. (1995) «Detecting Earnings Management », The Accounting Review 70(2) : 193-225.

DeFond M. L., Jiambalvo J. (1993) «Factors Related to Auditor-Client Disagreements over Income-Increasing Accounting Methods », Contemporary Accounting Research 9 : 415-431.

Dumontier P. (2003) «Les manipulations comptables et la qualité de l'information communiquée aux investisseurs », Revue du financier 139 (Février) : 65-73.

Francis, J. R., Krishnan, J. (1999) «Accounting Accruals and Auditor Reporting Conservatism », Contemporary Accounting Research $16: 135-166$.

Francis, J. R., Maydew E. L., Sparks H. C. (1999) «The Role of Big 6 Auditors in the Credible Reporting of Accruals », Auditing: A Journal of Practice and Theory $18: 17-34$.

Frankel R. M., Johnson M. F., Nelson K. K. (2002) «The Relation between Auditors' Fees for Nonaudit Services and Earnings Management », The Accounting Review 77(Supplement) : 71-105.

Guay W. P., Kothari S. P., Watts R. L. (1996) «A Market-Based Evaluation of Discretionary Accruals Models », Journal of Accounting Research 34 (Supplement) : 83-105.

Guay W., Verrechia R. (2006) «Discussion of an Economic Framework for Conservative Accounting and Bushman and Piotroski », Journal of Accounting and Economics 42 : 149-165.

Ghosh A., Moon D. (2005) "Auditor Tenure and Perceptions of Audit Quality », The Accounting Review 80(2) : 585-612.

Healy P. M., Wahlen J. M. (1999) «A Review of the Earnings Management Literature and its Implications for Standard Setting », Accounting Horizons 13(4) : 365-383.

Healy P., Palepu K. G. (1993) «The Effect of Firms' Financial Disclosure Policies on Stock prices », Accounting Horizons $7: 1-11$.

Holthausen R. W. (1990) «Accounting Method Choice: Opportunistic Behavior, Efficient Contracting and Information Perspectives », Journal of Accounting and Economics $12:$ 207-218.

Janin R. (2002) «Les contenus informationnels respectifs du résultat net et des indicateurs de flux de trésorerie dans le contexte français », Comptabilité - Contrôle - Audit 8(2) : 69-85.

Johnson V. E., Khurana I. K., Reynolds J. K. (2002) "Audit-Firm Tenure and the Quality of Financial Reports », Contemporary Accounting Research 19(4) : 637-660. 
Jones J. (1991) «Earnings Management During Import Relief Investigations », Journal of Accounting Research 29 (Autumn) : 193-228.

Kim J.-B., Chung R., Firth M. (2003) «Auditor Conservatism, Asymmetric Monitoring and Earnings Management », Contemporary Accounting Research 20(2) : 323-359.

Klein, B. R., Leffler, K. (1981) «The Role of Market Forces in Assuring Contractual Performance », Journal of Political Economy 89(4): 615-641.

Knapp M. C. (1991) «Factors that Audit Committee Members Use as Surrogates for Audit Quality », Auditing: A Journal of Practice and Theory 10(1) : 35-52.

Kothari S. P. (1992) «Price-Earnings Regressions in the Presence of Prices Leading Earnings: Earnings Level Versus Change Specification and Alternative Deflators », Journal of Accounting and Economics 15 : $173-202$.

Krishnan G. P. (2003) «Audit Quality and the Pricing of Discretionary Accruals », Auditing: A Journal of Practice and Theory 22(1) : 109-126.

Lin J. W., Li J. F., Yang J. S. (2006) «The Effect of Audit Committee Performance on Earnings Quality », Managerial Auditing Journal 21(9) : 921-933.

Menon K., Williams D. D. (2004) «Former Audit Partners and Abnormal Accruals », The Accounting Review 79 : 1095-1118.

Myers J. N., Myers L. A., Omer T. C. (2003) «Exploring the Term of the Auditor-Client Relationship and the Quality of Earnings: A Case for Mandatory Auditor Rotation? », The Accounting Review 78(3) : 779-799.

Nagy A. L. (2005) «Mandatory Audit Firm Turnover, Financial Reporting Quality, and Client Bargaining Power: The Case of Arthur Andersen », Accounting Horizons 19(2) : 51-68.

Obert R. (2006). Pratique des normes IFRS : Comparaison avec les règles françaises et les US GAAP, Dunod.

Petra S. T. (2007) « The Effects of Corporate Governance on the Informativeness of Earnings », Economics of Governance, forthcoming.

Piot C. (2004) «The Existence and Independence of Audit Committees in France », Accounting and Business Research 34(3) : 223-246.

Piot C., Janin R. (2007) «External Auditors, Audit Committees and Earnings Management in France », European Accounting Review 16(2) : 429-454.

Reynolds J. K., Deis Jr D. R., Francis J. R. (2004) «Professional Service Fees and Auditor Objectivity», Auditing: A Journal of Practice and Theory 23(1) : 29-52.

Subramanyan K. R. (1996) "The Pricing of Discretionary Accruals », Journal of Accounting and Economics $22: 249-281$.

Teoh S. H., Wong T. J. (1993) «Perceived Auditor Quality and the Earnings Response Coefficient », The Accounting Review 68(2) : 346-366.

Viénot M. (1995), «Le conseil d'administration des sociétés cotées », Document Association Française des Entreprises Privées (AFEP) \& Conseil National du Patronat Français (CNPF), juillet, Paris.

Viénot M. (1999), «Rapport du comité sur le gouvernement d'entreprise présidé par M. Marc Viénot», Document Association Française des Entreprises Privées (AFEP) \& Mouvement des Entreprises de France (MEDEF), juillet, Paris.

Watts R. L., Zimmerman J. L. (1986). Positive Accounting Theory, Englewood Cliffs, N. J. : Prentice-Hall.

Watts R. L., Zimmerman J. L. (1990) « Positive Accounting Theory: A Ten Year Perspective», The Accounting Review 65 : 131-156.

Webster E., Thornton D. B. (2004) « Earnings Quality under Rules vs. Principle-Based Accounting Standards: A Test of the Skinner Hypothesis », Working paper Queen's University.

Wild J. J. (1994) « Managerial Accountability to Shareholders: Audit Committees and the Explanatory Power of Earnings for Returns », British Accounting Review 26 : 353-374.

Wild J. J. (1996) «The Audit Committee and Earnings Quality », Journal of Accounting, Auditing and Finance $11(2): 247-276$. 
Tableau 1. Procédure d'obtention de l'échantillon

\begin{tabular}{|lc|}
\hline Étapes de la procédure d'obtention de l'échantillon de travail & $\begin{array}{c}\text { Nombre de } \\
\text { firmes restant }\end{array}$ \\
\hline Liste des sociétés du SBF 120 d'après site $\underline{w w w . c o b}$.fr au 17-12-2002 & 116 \\
Moins : sociétés financières, d'assurance, d'investissement & 103 \\
Moins : sociétés foncières et immobilières & 100 \\
Plus : sociétés (hors secteurs ci-dessus) entrées dans l'indice entre 1998 et 2002 et & 123 \\
non comprises dans liste de départ & 118 \\
Moins : élimination pour création ou introduction en bourse trop récente (2001, 2002) \\
$\begin{array}{l}\text { ou accès impossible à au moins un rapport annuel pour les exercices 1999-2001 } \\
\text { Moins : sociétés non référencées ou ne publiant pas de comptes consolidés dans }\end{array}$ \\
$\begin{array}{l}\text { DIANE } \\
\text { Moins : sociétés non rattachables à un secteur d'activité bien défini }\end{array}$ & 106 \\
\hline
\end{tabular}

Tableau 2. Mesures d'accruals - Statistiques descriptives sur trois ans (1999-2001)

\begin{tabular}{|l|c|c|c|c|c|c|c|c|}
\hline $\begin{array}{l}\text { Mesures } \\
\text { d'accruals }\end{array}$ & $\mathrm{N}$ & Moyenne & Médiane & $\begin{array}{c}\text { Écart } \\
\text {-type }\end{array}$ & Min & Max & $\begin{array}{c}\text { Nullité } \\
(t \text {-stat })\end{array}$ & $\begin{array}{c}\text { Diff. } \\
\text { Annuelle } \\
(f \text {-stat })\end{array}$ \\
\hline TA & 293 & $-0,020$ & $-0,033$ & 0,124 & $-0,380$ & 0,700 & $-2,708^{* *}$ & 1,072 \\
\hline D_TA & 262 & 0,022 & 0,008 & 0,136 & $-0,338$ & 0,828 & $2,629^{* *}$ & 2,697 \\
\hline AD_TA & 262 & 0,086 & 0,048 & 0,108 & 0,000 & 0,828 & & 2,828 \\
\hline
\end{tabular}

TA désigne les accruals totaux (Total Accruals). Le préfixe $\mathrm{D}_{-}$indique la composante discrétionnaire des accruals désignés. Le préfixe $\mathrm{AD}$ _désigne la valeur absolue des accruals discrétionnaires.

$*, * *$, et *** désignent une probabilité critique bilatérale inférieure à $0,05,0,01$ et 0,001 respectivement. 
Tableau 3. Statistiques descriptives selon la qualité du processus d'audit externe

\begin{tabular}{|c|c|c|c|c|c|c|}
\hline \multicolumn{7}{|c|}{ 1. Réputation de l'auditeur externe } \\
\hline & \multicolumn{2}{|c|}{$\operatorname{Big} N(n=122)$} & \multicolumn{2}{|c|}{ Autres $(\mathrm{n}=30)$} & \multirow{3}{*}{$\begin{array}{l}t \text {-stat } \\
-2,279 \\
* * *\end{array}$} & \multirow{3}{*}{$\begin{array}{l}z \text {-stat } \\
-2,495 \\
* * *\end{array}$} \\
\hline & Moyenne & Médiane & Moyenne & Médiane & & \\
\hline RN & 0,050 & 0,043 & 0,074 & 0,074 & & \\
\hline TA & $-0,032$ & $-0,036$ & 0,003 & $-0,020$ & $-\underset{* *}{1,611}$ & $-1,065$ \\
\hline ABS TA & 0,073 & 0,053 & 0,094 & 0,068 & $-1,277$ & $-1,083$ \\
\hline $\mathrm{CFO}$ & 0,083 & 0,084 & 0,071 & 0,084 & 0,522 & $-0,125$ \\
\hline DAC & 0,008 & 0,007 & 0,057 & 0,016 & $-1,579$ & $-0,963$ \\
\hline NDAC & $-0,040$ & $-0,032$ & $-0,053$ & $-0,032$ & 0,946 & $-0,148$ \\
\hline ABS DAC & 0,069 & 0,040 & 0,115 & 0,067 & $\begin{array}{l}-1,973 \\
* *\end{array}$ & $\begin{array}{c}-2,143 \\
* *\end{array}$ \\
\hline \multicolumn{7}{|c|}{ 2. Durée (T) du mandat de l'auditeur externe } \\
\hline & \multicolumn{2}{|c|}{$\mathrm{T}>6$ ans $(\mathrm{n}=87)$} & \multicolumn{2}{|c|}{$\mathrm{T} \leq 6$ ans $(\mathrm{n}=47)$} & & \\
\hline $\mathrm{RN}$ & $\begin{array}{c}\text { Moyenne } \\
0,046\end{array}$ & $\begin{array}{c}\text { Médiane } \\
0,042\end{array}$ & $\begin{array}{c}\text { Moyenne } \\
0,055\end{array}$ & $\begin{array}{c}\text { Médiane } \\
0,053\end{array}$ & $\begin{array}{l}t \text {-stat } \\
-0,986\end{array}$ & $\begin{array}{c}z \text {-stat } \\
-1,494 \\
*\end{array}$ \\
\hline TA & $-0,020$ & $-0,024$ & $-0,042$ & $-0,041$ & 1,190 & $-1,215$ \\
\hline ABS TA & 0,076 & 0,058 & 0,072 & 0,048 & 0,320 & $-0,333$ \\
\hline $\mathrm{CFO}$ & 0,066 & 0,063 & 0,097 & 0,100 & $\begin{array}{c}-1,621 \\
*\end{array}$ & $\begin{array}{c}-1,979 \\
* *\end{array}$ \\
\hline DAC & 0,020 & 0,010 & 0,016 & 0,004 & 0,179 & $-0,427$ \\
\hline NDAC & $-0,039$ & $-0,031$ & $-0,058$ & $-0,043$ & 1,235 & $\begin{array}{c}-1,504 \\
*\end{array}$ \\
\hline ABS DAC & 0,077 & 0,048 & 0,076 & 0,040 & 0,017 & $-0,100$ \\
\hline
\end{tabular}

RN désigne le résultat net, TA les accruals totaux, ABS TA les accruals totaux en valeur absolue, CFO le flux de trésorerie opérationnel, DAC les accruals discrétionnaires, NDAC les accruals non discrétionnaires, ABS DAC les accruals discrétionnaires en valeur absolue.

t-stat : $\mathrm{t}$ de student pour la comparaison des moyennes de deux échantillons indépendants (corrigé pour l'hétérogénéité de la variance le cas échéant).

$z$-stat : le test non paramétrique de Mann-Whitney porte sur l'hypothèse nulle d'homogénéité des distributions.

$*, * *$ et $* * *$ désignent une probabilité critique unilatérale inférieure à $10 \%, 5 \%$ et $1 \%$ respectivement. 
Tableau 4. Statistiques descriptives selon les caractéristiques du comité d'audit

\begin{tabular}{|c|c|c|c|c|c|c|c|c|c|c|c|c|c|c|c|c|c|c|}
\hline & \multicolumn{2}{|c|}{$\begin{array}{l}\text { A. Présence d'un } \\
\text { comité d'audit }\end{array}$} & \multicolumn{2}{|c|}{$\begin{array}{l}\text { B. Comité d'audit } \\
\text { indépendant }\end{array}$} & \multicolumn{2}{|c|}{$\begin{array}{l}\text { C. Pas de comité ou } \\
\text { comité d'audit non } \\
\text { indépendant }\end{array}$} & \multicolumn{2}{|c|}{$\begin{array}{c}\text { D. Pas de comité } \\
\text { d'audit }\end{array}$} & \multicolumn{2}{|c|}{$\begin{array}{l}\text { E. Comité d'audit } \\
\text { non indépendant }\end{array}$} & \multicolumn{2}{|c|}{ A. vs D. } & \multicolumn{2}{|c|}{ B. vs C. } & \multicolumn{2}{|c|}{ B. vs D. } & \multicolumn{2}{|c|}{ B. vs E. } \\
\hline & $(\mathrm{n}=$ & 100) & $(n=$ & 56) & $(n=$ & 89) & $(\mathrm{n}=$ & 50) & $(\mathrm{n}=$ & 39) & & & & & & & & \\
\hline $\mathrm{RN}$ & $\begin{array}{c}\text { Moyenne } \\
0,047\end{array}$ & $\begin{array}{c}\text { Médiane } \\
0,042\end{array}$ & $\begin{array}{c}\text { Moyenne } \\
0,045\end{array}$ & $\begin{array}{c}\text { Médiane } \\
0,044\end{array}$ & $\begin{array}{c}\text { Moyenne } \\
0,063\end{array}$ & $\begin{array}{l}\text { Médiane } \\
0,053\end{array}$ & $\begin{array}{c}\text { Moyenne } \\
0,074\end{array}$ & $\begin{array}{l}\text { Médiane } \\
0,068\end{array}$ & $\begin{array}{c}\text { Moyenne } \\
0,049\end{array}$ & $\begin{array}{c}\text { Médiane } \\
0,035\end{array}$ & $\begin{array}{l}t-s t a t \\
-3,180 \\
* * *\end{array}$ & $\begin{array}{c}z-\text {-stat } \\
-3,181 \\
* * *\end{array}$ & $\begin{array}{l}t-s t a t \\
-2,084 \\
* * *\end{array}$ & $\begin{array}{c}z \text {-stat } \\
-1,381 \\
*\end{array}$ & $\begin{array}{l}t-s t a t \\
-3,128 \\
* * *\end{array}$ & $\begin{array}{c}z-s t a t \\
-2,797 \\
* * *\end{array}$ & $\begin{array}{l}t \text {-stat } \\
-0,375\end{array}$ & $\begin{array}{l}z \text {-stat } \\
-0,772\end{array}$ \\
\hline TA & $-0,042$ & $-0,040$ & $-0,032$ & $-0,034$ & $-0,022$ & $-0,025$ & 0,008 & $-0,014$ & $-0,061$ & $-0,055$ & $\begin{array}{r}-2,709 \\
* * *\end{array}$ & $\begin{array}{r}-2,161 \\
* *\end{array}$ & $-0,534$ & $-0,223$ & $\begin{array}{r}-1,882 \\
* *\end{array}$ & $\begin{array}{r}-1,430 \\
*\end{array}$ & $\begin{array}{r}1,412 \\
*\end{array}$ & $\begin{array}{r}-1,294 \\
*\end{array}$ \\
\hline TA ABS & 0,077 & 0,057 & 0,073 & 0,053 & 0,080 & 0,055 & 0,077 & 0,048 & 0,083 & 0,061 & $-0,012$ & $-0,702$ & $-0,492$ & $-0,037$ & $-0,270$ & $-0,335$ & $-0,648$ & $-0,469$ \\
\hline $\mathrm{CFO}$ & 0,090 & 0,086 & 0,078 & 0,081 & 0,086 & 0,087 & 0,066 & 0,084 & 0,110 & 0,103 & 1,221 & $-0,458$ & $-0,421$ & $-1,015$ & 0,539 & $-0,247$ & $\begin{array}{r}-1,399 \\
*\end{array}$ & $-1,596$ \\
\hline DAC & $-0,005$ & $-0,001$ & 0,008 & 0,003 & 0,024 & 0,017 & 0,062 & 0,023 & $-0,025$ & $-0,002$ & $\begin{array}{r}-2,885 \\
* * *\end{array}$ & $\begin{array}{r}-2,978 \\
* * *\end{array}$ & $-0,770$ & $-1,198$ & $\begin{array}{r}-2,250 \\
* * *\end{array}$ & $\begin{array}{r}-2,424 \\
* * *\end{array}$ & $\begin{array}{r}1,598 \\
*\end{array}$ & $-0,666$ \\
\hline NDAC & $-0,038$ & $-0,031$ & $-0,040$ & $-0,030$ & $-0,046$ & $-0,036$ & $-0,054$ & $-0,036$ & $-0,036$ & $-0,039$ & $\begin{array}{r}1,434 \\
*\end{array}$ & $\begin{array}{r}-1,435 \\
*\end{array}$ & 0,519 & $-1,170$ & 0,982 & $\begin{array}{r}-1,462 \\
*\end{array}$ & $-0,376$ & $-0,431$ \\
\hline ABS_DAC & 0,067 & 0,041 & 0,061 & 0,035 & 0,089 & 0,044 & 0,101 & 0,053 & 0,073 & 0,041 & $\begin{array}{r}-1,788 \\
* * *\end{array}$ & $\begin{array}{r}-1,340 \\
*\end{array}$ & $\begin{array}{r}-1,918 \\
* *\end{array}$ & $\begin{array}{r}-1,446 \\
*\end{array}$ & $\begin{array}{r}-2,045 \\
* * *\end{array}$ & $\begin{array}{r}-1,633 \\
*\end{array}$ & $-0,789$ & $-0,741$ \\
\hline
\end{tabular}

RN désigne le résultat net, TA les accruals totaux, ABS TA les accruals totaux en valeur absolue, CFO le flux de trésorerie opérationnel, DAC les accruals discrétionnaires, NDAC les accruals non discrétionnaires, ABS DAC les accruals discrétionnaires en valeur absolue.

$t$-stat : $\mathrm{t}$ de student pour la comparaison des moyennes de deux échantillons indépendants (corrigé pour l'hétérogénéité de la variance le cas échéant).

$z$-stat : le test non paramétrique de Mann-Whitney porte sur l'hypothèse nulle d'homogénéité des distributions.

$*, * *$ et $* * *$ désignent une probabilité critique unilatérale inférieure à $10 \%, 5 \%$ et $1 \%$ respectivement. 
Tableau 5. Régressions linéaires des rentabilités sur les flux de trésorerie opérationnel, accruals non discrétionnaires et accruals discrétionnaires conditionnés par la qualité de l'audit $(\mathrm{N}=152$ firmes-année)

$\mathrm{RETt}=\beta 0+\beta 1 \mathrm{CFOt}+\beta 2 \mathrm{DACt}+\beta 3 \mathrm{NDACt}+\beta 4 \mathrm{Xt} * \mathrm{DACt}+\beta 5 \mathrm{Xt}+\beta 6 \mathrm{Y} 1999+\beta 7 \mathrm{Y} 2000+\varepsilon$

\begin{tabular}{|c|c|c|c|c|c|c|c|c|c|}
\hline & \multicolumn{2}{|c|}{$X=\operatorname{Big} 5$} & \multicolumn{2}{|c|}{$\mathrm{X}=$ Tenure } & \multicolumn{2}{|c|}{$\mathrm{X}=\mathrm{AC}$} & \multicolumn{2}{|c|}{$\mathrm{X}=\mathrm{ACIND}$} \\
\hline & & coef. & $t$-stat & coef. & $t$-stat & coef. & t-stat & coef. & $t$-stat \\
\hline cte & & $-0,251$ & $-4,447 * * *$ & $-0,175$ & $-3,399 * * *$ & $-0,248$ & $-4,783 * * *$ & $-0,231$ & $-5,315 * * *$ \\
\hline $\mathrm{CFO}$ & $(+)$ & 2,770 & $4,939 * * *$ & 2,934 & $4,591 * * *$ & 2,520 & $4,292 * * *$ & 2,425 & $4,129 * * *$ \\
\hline DAC & $(+)$ & 3,942 & $5,336 * * *$ & 3,299 & $4,567 * * *$ & 3,083 & $4,220 * * *$ & 2,822 & $4,422 * * *$ \\
\hline NDAC & $(+)$ & 2,967 & $4,427 * * *$ & 3,327 & $4,325 * * *$ & 2,782 & $3,854 * * *$ & 2,612 & $3,684 * * *$ \\
\hline $\mathrm{X} * \mathrm{DAC}$ & $(-)$ & $-1,199$ & $-2,487 * *$ & $-0,172$ & $-0,355$ & $-0,503$ & $-1,100$ & $-0,633$ & $-1,605 *$ \\
\hline $\mathrm{X}$ & $?$ & 0,032 & 0,583 & $-0,049$ & $-0,983$ & 0,047 & 0,965 & 0,060 & 1,305 \\
\hline Y1999 & $?$ & 0,192 & $3,200 * * *$ & 0,201 & $3,086 * * *$ & 0,206 & $3,375 * * *$ & 0,213 & $3,457 * * *$ \\
\hline Y2000 & $?$ & 0,059 & 1,154 & 0,049 & 0,872 & 0,057 & 1,106 & 0,050 & 0,945 \\
\hline
\end{tabular}

CFO désigne le flux de trésorerie opérationnel, DAC les accruals discrétionnaires, NDAC les accruals non discrétionnaires, BIG5 : variable binaire codée 1 si au moins un des Commissaires aux comptes titulaires fait partie des Big Five, et 0 sinon, Tenure : variable binaire codée 1 si le nombre d'années de fonction du CAC dominant et supérieur à 6 et 0 sinon, $\mathrm{AC}$ : variable binaire codée 1 si la société a mis en place un comité d'audit et 0 sinon, ACIND : variable binaire codée 1 le comité d'audit est composé d'au moins $50 \%$ de membres indépendants.

$*$, ** et $* * *$ désignent une probabilité critique bilatérale inférieure à $10 \%, 5 \%$ et $1 \%$ respectivement (unilatérale pour $\mathrm{X} * \mathrm{DAC}$ ).

Tableau 6. Régressions linéaires des rentabilités sur les flux de trésorerie opérationnel, accruals non discrétionnaires et accruals discrétionnaires conditionnés simultanément par la réputation de l'auditeur externe et l'indépendance du comité d'audit ( $N=145$ firmes-année)

\begin{tabular}{|c|c|c|c|c|c|c|c|c|c|c|}
\hline & cte & $\begin{array}{c}\mathrm{CFO} \\
(+)\end{array}$ & $\begin{array}{c}\text { DAC } \\
(+)\end{array}$ & $\begin{array}{c}\text { NDAC } \\
(+)\end{array}$ & $\begin{array}{c}\text { BIG5*DAC } \\
(-)\end{array}$ & $\begin{array}{c}\text { BIG5 } \\
?\end{array}$ & $\begin{array}{c}\text { ACIND*DAC } \\
(-)\end{array}$ & $\begin{array}{c}\text { ACIND } \\
?\end{array}$ & $\begin{array}{c}\text { Y1999 } \\
?\end{array}$ & $\begin{array}{c}\text { Y2000 } \\
?\end{array}$ \\
\hline coef. & $-0,245$ & 2,589 & 3,727 & 2,762 & $-1,078$ & 0,008 & $-0,361$ & 0,057 & 0,201 & 0,052 \\
\hline$t$-stat & $\begin{array}{c}-4,053 \\
\quad * * *\end{array}$ & $\begin{array}{c}4,409 \\
* * *\end{array}$ & $\begin{array}{c}4,833 \\
* * *\end{array}$ & $\begin{array}{c}3,904 \\
* * *\end{array}$ & $\begin{array}{c}-2,076 \\
* *\end{array}$ & 0,137 & $-0,878$ & 1,232 & $\begin{array}{c}3,275 \\
* * *\end{array}$ & 0,987 \\
\hline
\end{tabular}

CFO désigne le flux de trésorerie opérationnel, DAC les accruals discrétionnaires, NDAC les accruals non discrétionnaires, BIG5 : variable binaire codée 1 si au moins un des Commissaires aux comptes titulaires fait partie des Big Five, et 0 sinon, ACIND : variable binaire codée 1 le comité d'audit est composé d'au moins $50 \%$ de membres indépendants.

$*$, ** et *** désignent une probabilité critique bilatérale inférieure à $10 \%, 5 \%$ et $1 \%$ respectivement. 
${ }^{\mathrm{i}}$ Comme le souligne Robert Obert (2004), « même si l'IASB considère que les informations fournies par les états financiers peuvent être utiles à toutes les personnes en relation avec l'entreprise, il semble bien qu'une présentation orientée vers les besoins des investisseurs ait été privilégiée ». Pour le moins, le fait que le cadre conceptuel de l'IASB classe les investisseurs en premier dans leur liste des utilisateurs de la comptabilité n'est sans doute pas innocent.

${ }^{\text {ii }}$ Voir notamment Becker et al. (1998), Butler et al. (2004), Menon et Williams (2004).

${ }^{i i i}$ Les sociétés financières ont une réglementation spécifique en termes de présentation des états financiers, de gouvernance et d'audit. À titre d'exemple, les comités d'audit sont requis et soumis à un formalisme élevé, et les auditeurs externes doivent être agréés par la Commission Bancaire.

iv À titre d'exemple, Dechow (1994) exclut de son échantillon, les entreprises dont le flux de trésorerie opérationnel et le résultat se situent au-delà du $99^{\text {ème }}$ centile.

${ }^{v}$ Afin d'apprécier la sensibilité de nos résultats aux problèmes de mesure des accruals discrétionnaires, nous avons également appliqué deux autres approches du modèle de Jones : l'approche traditionnelle et la version consistant à enrichir le modèle précédent de l'effet des flux de trésorerie opérationnel, en intégrant cette variable au niveau de l'estimation même des paramètres.

${ }^{\mathrm{vi}}$ Les variables comptables (CFO, DAC, NDAC) sont normées par le cours de bourse de début de période. Christie (1987) ou Kothari (1992) ont montré que le fait de normer les variables comptables par le prix du titre en début de période améliore la spécification des modèles et minimise l'impact de l'hétéroscédasticité des résidus.

${ }^{\text {vii }}$ Certains travaux empiriques anglo-saxons (Johnson et al., 2002 ; Nagy, 2005) qualifient de « longue » une collaboration auditeur-audité strictement supérieure à huit années. Dans le cadre français, où le mandat est donné pour six exercices financiers, renouvelable sans limitation de durée, on peut alors considérer comme « longue » une collaboration strictement supérieure à six ans. Autrement dit, une fois le premier renouvellement acquis, l'auditeur est considéré comme « enraciné ». 